\title{
Damping Behaviour Analysis of 3D Printed Samples with Pores
}

\author{
J. GÖKEN ${ }^{a, *}$ AND N. SABA ${ }^{b}$ \\ ${ }^{a}$ University of Applied Sciences Emden/Leer, Faculty of Maritime Sciences, \\ Bergmannstr. 36, 26789 Leer, Germany \\ ${ }^{b}$ University of Balamand, Faculty of Engineering, Department of Mechanical Engineering, \\ Koura campus, 100 P.O. Box Tripoli, Lebanon
}

Received: 05.07.2020 \& Accepted: 31.08.2020

Doi: 10.12693/APhysPolA.138.844 *e-mail: juergen.goeken@hs-emden-leer.de

\begin{abstract}
3D printing offers the possibility of introducing pores into the material in a targeted manner. This results in a low-density material which can also act as a passive damping material with increased damping properties. Against the background of noise and vibration control, an adjustable damping behaviour is of technical interest. Strain- and frequency-dependent damping measurements were carried out at room temperature on rectangular samples made from polylactic acid which is a bioactive and biodegradable polyester. The samples were produced using a commercially available 3D printer. Different pore sizes and pore distances were realised. Afterwards, the impact on the damping behaviour was analysed. A monotonic increase in damping of both non-porous and porous specimens occured when the increase of material strain was noticed. The damping was dependent on the pore size but this became clear for larger pores. Pore spacing seemed to be less important. Frequency-dependent damping measurements of non-porous and porous samples showed a slight decrease in damping with the increasing frequency in the selected frequency range from $10 \mathrm{~Hz}$ to about $60 \mathrm{~Hz}$. The results were significantly influenced by the pore size. A slight increase in material damping with the increasing pore spacing was observed. Further, "voids" (empty spaces) were found in the immediate vicinity around the pores which varied greatly in both length and width. Their individual influence on the overall damping must be taken into account. Only after a certain combination of pore size and porosity onwards, a dominant influence of these two parameters can be expected.
\end{abstract}

topics: 3D printing, polylactic acid, pores, damping measurement

\section{Introduction}

The layer-by-layer manufacturing process of $3 \mathrm{D}$ printing became essential in the field of prototype production. The main advantage of this technique is the reduction of prototypes' fabrication time which results in a significant shortening of the total time needed for product development [1]. In addition, this technique is also currently used in tools production as well as in series and spare parts production [2].

In medicine, not only prostheses are now 3D printed at low prices [3] but also organs, tissues and precise tissue cultures — such as organ parts made from collagen [4] — are produced in this way. Recently, a special 3D printed concrete has been invented that could revolutionise the construction industry [5].

The 3D printing offers the possibility of introducing pores into the material in a targeted manner. The advantages are weight-reduction through hollow structures and, thus, the production of complex geometries without the need of costly assembly processes. The disadvantage is the reduced values of mechanical properties, such as impact resistance and flexibility of $3 \mathrm{D}$ printed products [6]. This is a direct result of the materials used in this technique which are currently only substituted materials.

\section{Method}

\subsection{Noise and vibration control by cellular materials}

Many physical and mechanical properties of materials are correlated with the density. The Young modulus together with the density determines most of the acoustical properties of a cellular material like wood, as shown by Wegst [7]. Porous materials are believed in general to reduce noise. According to Göransson [8], porous solid may be characterised as an elastic-viscoelastic and acoustic-viscoacoustic medium.

Passive damping is one of the most effective and widely used methods for noise and vibration control. This offers the greatest advantages when used to reduce the amplitude of dynamic instabilities or resonances in a structure. It is therefore not surprising that the transport sector, e.g. the shipping 


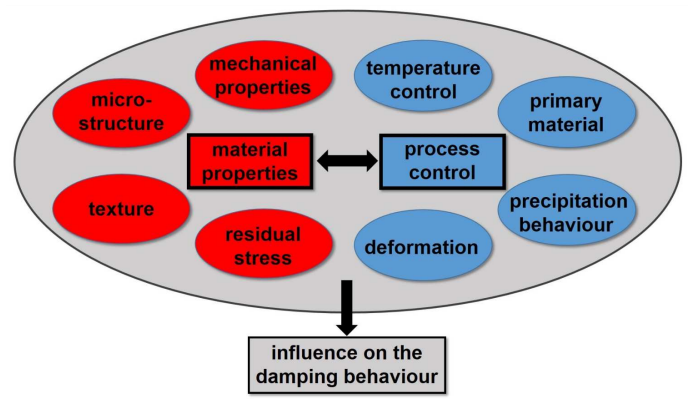

Fig. 1. Impacts on material damping.

sector, is looking for materials or components that are characterised by high noise absorption. In order to decrease noise emission for the personnel on board of a vessel and the emission of machine noise to the environment, especially under water, undesirable vibrations in a structure are to be reduced. This is fundamental because anthropogenic sound emitted to the marine environment can affect marine organisms in a number of ways. This sound can mask biologically relevant signals so that it can lead to a variety of abnormal behavioural reactions. Furthermore, hearing organs can be affected in the form of a hearing loss and at very high reception levels; sound can injure or even kill marine life [9]. Therefore, material analysis of modern hull and sailing material becomes an important task in the shipping sector. This objective should be pursued for reducing noise emissions emanating from a seagoing vessel.

Noise and vibrations could be controlled by means of a vibration insulating (damping) layer. Cost and time reduction of such components (e.g. sound-absorbing panels), which assure protective measures for vessel personnel, as well as solving individual construction problems, can be realised by using the $3 \mathrm{D}$ printing process. Growth of a further demand in key end-use industries, such as consumer goods, healthcare and transportation, is expected to remain the key driving force for the global 3D printing market [10].

The damping behaviour of materials can be influenced by several parameters (see Fig. 1). Process control and material properties interact with each other. In this context, 3D printing becomes essential for the production of samples for damping measurements with exact same dimensions and/or equal microstructures due to its advantage of high process reproducibility.

It is well known that foamed materials often exhibit higher damping properties than the corresponding compact materials [11]. Contrary to other mechanical characteristics, only little attention is paid to mechanical damping of foams and other cellular metallic materials. Cell shape, volume fraction of solid contained in the cell edges, sphericity, connectivity factor and morphological factor (cell elongation) can influence the mechanical properties

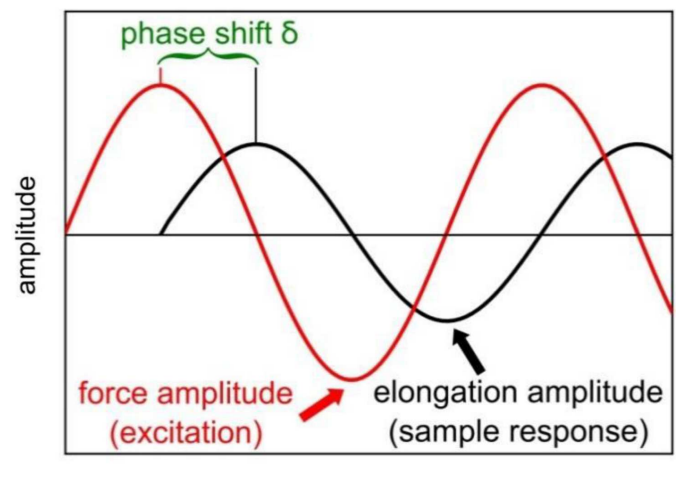

time

Fig. 2. Sinusoidal oscillation of the exciting force and sample response of a viscoelastic material.

of cellular metallic materials [12]. Density, pore size, chemical composition, pre-deformation and time of vibration are parameters that can affect the amplitude-dependent damping of foams [13].

As emphasised by Han et al. [14], many articles reported that the damping capacity of porous materials increased as the porosity increased and, generally, at a rate several times higher than that of the parent materials. Therefore, it is not clear if the porosity acts as a dominant parameter for materials' damping. The use of a 3D printer allows the production of samples with the same dimensions but with pores' different distance and size.

\subsection{Vibration damping}

Theoretical foundations for the calculation of material damping were introduced by Lazan [15]. For an applied stress due to an external force varying sinusoidally with time, a viscoelastic material will consequently respond with a corresponding sinusoidal strain for low amplitudes of stress. The sinusoidal variation in time is usually described as a rate specified by the excitation frequency ( $\omega$ is angular frequency). The strain $\varepsilon$ of a viscoelastic body is out of phase with the applied stress $\sigma$, leading to the phase angle $\delta$, see Fig. 2. This phase lag is due to the excess time which is required for the appearance of atomic or molecular motions and relaxations [16].

Damping can be measured in terms of the loss tangent as

$$
\tan (\delta)=\frac{E^{\prime \prime}}{E^{\prime}}
$$

where $E^{\prime}$ is the storage modulus which describes the ability of the material to store potential energy and release it upon deformation. The imaginary loss modulus $E^{\prime \prime}$ is associated with energy dissipation in the form of heat upon deformation. The combination of both moduli leads to the complex modulus of elasticity $E^{*}$ (the so-called dynamic modulus), namely

$$
E^{*}=E^{\prime}+\mathrm{i} E^{\prime \prime}
$$




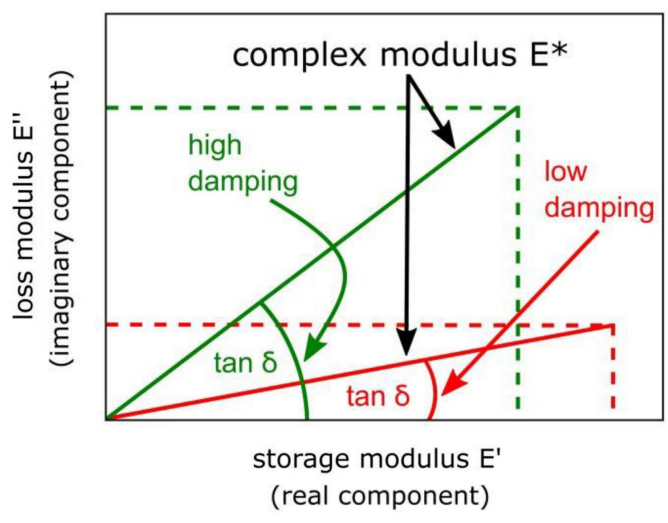

Fig. 3. Determination of the loss tangent $\tan (\delta)$ from the storage modulus $E^{\prime}$ and the loss modulus $E^{\prime \prime}$.

The loss tangent $\tan (\delta)$ becomes therefore a measure of the energy loss expressed in terms of the recoverable energy and it represents mechanical damping or internal friction in a viscoelastic system. Further explanations can be found, e.g. in [17].

Figure 3 shows the dependence of the loss tangent $\tan (\delta)$ on $E^{\prime}$ and $E^{\prime \prime}$. In the case of the dominance of the elastic behaviour of the material, the storage modulus $E^{\prime}$ is larger than the loss modulus $E^{\prime \prime}$ which leads to a small loss tangent. If $E^{\prime \prime}$ increases, then the viscous behaviour of the material becomes significant.

Referring to (1), it is obvious that $\tan (\delta)$ and $E^{\prime}$ are reciprocal to each other. For low damping materials, the storage modulus $E^{\prime}$ becomes dominant and is very similar to the Young modulus $E$.

\section{Experimental details}

In this work, strain- and frequency-dependent damping measurements on rectangular samples made from polylactic acid (PLA) were carried out. The samples are $3 \mathrm{D}$ printed and possess defined pore sizes and pore distance.

\subsection{Sample material}

PLA is a bioactive and biodegradable polyester. It consists of lactic acid building blocks. Most "bioplastic" products are not made of a pure bio-based plastic but of mixtures of different types of plastic, the so-called blends. This allows for having a product made, e.g., in $70 \%$ of bio-based materials, while the remaining $30 \%$ is a conventional mineral oilbased plastic. For instance, bioplastics are made on a starch basis which is mixed with a water-repellent conventional polymer.

The bio-based plastics can be further divided into drop-in solutions and new types of biopolymers. The bio-based chemically novel polymers that are not structurally identical to conventional plastics include, e.g. PLA. The PLA has good mechanical properties, such as high surface hardness, rigidity and a high modulus of elasticity (tensile strength)
Properties of PLA (typical values).

TABLE I

\begin{tabular}{l|c}
\hline \hline \multicolumn{1}{c|}{ Property } & Value \\
\hline type of polymer [19] & thermoplastic \\
physical state [19] & solid \\
density $\left[\mathrm{g} / \mathrm{cm}^{3}\right][19]$ & $1.21-1.25$ \\
melting point $\left[{ }^{\circ} \mathrm{C}\right][19]$ & $150-162$ \\
glass temperature $\left[{ }^{\circ} \mathrm{C}\right][19]$ & $45-60$ \\
notched izod impact strength $[\mathrm{J} / \mathrm{m}][20]$ & 26 \\
water absorption [\%] [21] & $\approx 1$ \\
tensile strength [MPa] [20] & 59 \\
elongation at break [\%] [20] & 7 \\
molar mass $M_{n}[\mathrm{~g} / \mathrm{mol}][22]$ & $\approx 70000$ \\
molecular formula [23] & $\left(\mathrm{C}_{3} \mathrm{H}_{4} \mathrm{O}_{2}\right)_{n}$
\end{tabular}

but only moderate impact resistance. The main properties of polylactic acid are shown in Table I. In fact, PLA can be used to produce deformable plastics (thermoplastics) [18].

Physical properties of PLA include low moisture absorption with a high capillary effect, low flammability as well as high UV resistance and colour fastness. In addition, PLA's density is relatively low which makes it suitable for lightweight applications. The flexural strength is about 100-118 MPa [24]. Due to the low melting point, as a result neither a heated print bed nor a closed chamber is necessary when printing with PLA.

The PLA filament retains the typical properties of PLA in a slightly modified form. It shows higher resistance and less brittleness. Due to the low shrinkage factor, the PLA will no longer deform after cooling, so that warping occurs only to a very small extent. Furthermore, the printing process is very simple and printing at low temperatures with low odour is possible. The used PLA filament (supplier: Velleman ${ }^{\circledR}$ nv, Gavere, Belgium) has a diameter of $1.75 \mathrm{~mm}$ and a relative density of $1.24 \mathrm{~g} / \mathrm{cm}^{3}$. The temperature for printing of the material is $180-210^{\circ} \mathrm{C}[25]$.

\section{2. $3 \mathrm{D}$ printing}

A commercially available 3D printer Creality CR20 was used. Device specifications can be found in [26]. The applied printing technology was fused deposition modelling (FDM). It describes a manufacturing process in which a workpiece is built up in layers from a meltable plastic like PLA.

In fused deposition modelling, the starting material is usually in the form of a plastic wire. This wire is also called filament. Wound on a coil, the filament is pushed through an extruder (also called the cold end) into the so-called hot end (similar to an inkjet print head) until the material is extruded from the nozzle. The molten filament is applied to a usually heated pressure bed. The filament is spread out in layers and cools down during the printing process so that it bonds with the filament of the next layer but does not fuse completely [27]. The sample 
preparation was carried out at a printing temperature of $200^{\circ} \mathrm{C}$, whereas the build plate temperature was $50^{\circ} \mathrm{C}$. A layer thickness of $0.16 \mathrm{~mm}$ and a print speed of $50 \mathrm{~mm} / \mathrm{s}$ were adjusted.

\subsection{Damping equipment}

Damping was measured with the help of a dynamic mechanical analyser (DMA, EPLEXOR ${ }^{\circledR}$ $500 \mathrm{~N}$ ). Dynamic mechanical analysis is a technique used to study and characterise materials with respect to material phase transitions and the response to mechanical and thermal stress. This is particularly applicable to viscoelastic materials.

DMA machines work under the concept of applying a force to a material and analysing the material's response to that force (a non-resonance method). The force used in this case is sinusoidal and oscillates at a range of frequencies and across a range of temperatures. From analysing this response, the DMA software is able to calculate various parameters from the recorded dynamic modulus $E^{*}[28,29]$. The DMA used is characterised by a high total force of $1500 \mathrm{~N}$. The dynamic force amplitude is $\pm 500 \mathrm{~N}$. A frequency range from 0.01 to $100 \mathrm{~Hz}$ can be covered. Depending on the goal and type of experiment, parameters such as static preload, amplitude and frequency of dynamic loading can be defined. For more information on the DMA specifications, see [30].

The samples were fixed at both ends, as well as in the middle. They were statically and dynamically loaded in the middle using a dual cantilever holder. A dynamic force is superimposed on the static force which results in a dynamic distortion of the sample.

The contact force for sample clamping was set to $2.0 \mathrm{~N}$ (accuracy $\approx \pm 0.3 \mathrm{~N}$ ). The dynamic load is based on the chosen material's strain range which has to be covered. The material strain $\varepsilon$ is obtained from $\varepsilon=\frac{\Delta h}{h}$, where $h$ is the thickness and $\Delta h$ is the maximum deflection of the sample. Here, the deflection strain is denoted by $\varepsilon$.

\subsection{Sample preparation}

The components were constructed in a CAD program. The CAD model was transferred to a standard triangle language (STL) format which is the most common standard interface between CAD and rapid prototyping or 3D printing [31]. Using this format, the surface of an object is described as a triangular mesh. The printing is an additive manufacturing process because the construction of a component arises through individual layers of material.

Each sample was produced with a length of $80.0 \mathrm{~mm}$, a width of $10.0 \mathrm{~mm}$ and a thickness of $3.0 \mathrm{~mm}$. The values of the length, width and thickness of the samples show a standard deviation of less than $11 \%$. Initially, three samples without pores were prepared in order to have a reference for the damping measurements. Subsequently, additional samples were printed with the same pore spacing

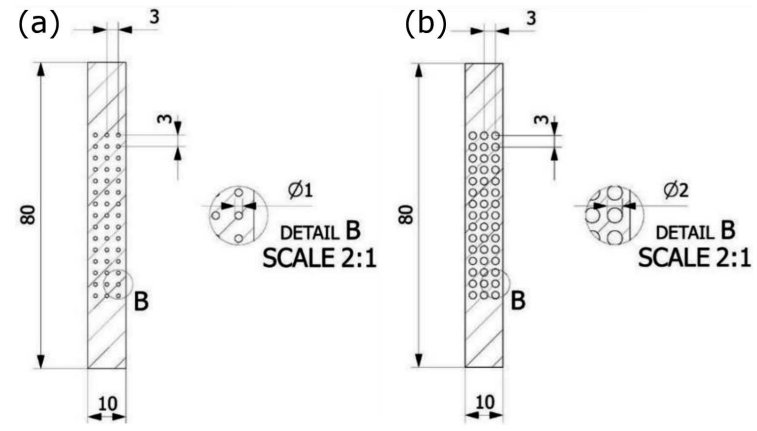

Fig. 4. Sample with (a) $1 \mathrm{~mm}$ and (b) $2 \mathrm{~mm}$ pore size. The distance from the centre of a pore to the centre of a pore has remained the same with $3 \mathrm{~mm}$.

TABLE II

Variation of pore diameter (pore distance: $3 \mathrm{~mm}$, pores per sample: 45$)$.

\begin{tabular}{c|c}
\hline \hline $\begin{array}{c}\text { Diameter of } \\
\text { the pores }[\mathrm{mm}]\end{array}$ & Porosity [\%] \\
\hline 1.0 & 1.0 \\
1.2 & 1.8 \\
1.4 & 2.8 \\
1.6 & 4.2 \\
1.8 & 6.0 \\
2.0 & 8.2
\end{tabular}

but different pore diameters. The distance of $3 \mathrm{~mm}$ from a pore centre to a pore centre remained the same for every pore size.

Figure 4 shows the CAD models of two samples, one with the smallest pore size (see Fig. 4a) and one with the largest pore size (see Fig. 4b). Based on the pore diameter and constant sample dimension, the porosity $c$ can be calculated as

$$
c=\frac{V_{P, \text { tot }}}{V_{\mathrm{tot}}}
$$

where $V_{P \text {, tot }}$ is the total pore volume and $V_{\text {tot }}$ is the total volume of the non-porous sample. The different pore diameters and porosity [\%] are listed in Table II. The porosity values were calculated according to (3). A maximum tolerance of about $\pm 20 \%$ of the shown porosity data has to be considered.

In further measurements, the pore size was kept constant but the pore spacing was changed. The pore size was $1.4 \mathrm{~mm}$ and the pore spacing was increased from $3.0 \mathrm{~mm}$ to $3.8 \mathrm{~mm}$ (see Table III). Due to varying pore distances, less pores per sample could be printed.

Strain-dependent damping measurements at a constant measuring frequency of $50 \mathrm{~Hz}$ were carried out at a range of the dynamic load ranging from $0.3 \%$ of the sample thickness $h$ to about $20 \%$, whereas the dynamic force was generated by an electrodynamic shaker system. An incremental rise of the dynamic force of $2 \mathrm{~N}$ was applied. Additionally, 
TABLE III

Variation of pore distance (diameter of the pores is $1.4 \mathrm{~mm}$ ).

\begin{tabular}{c|c|c}
\hline \hline $\begin{array}{c}\text { Pore } \\
\text { distance }[\mathrm{mm}]\end{array}$ & $\begin{array}{c}\text { Pores } \\
\text { per sample }\end{array}$ & Porosity [\%] \\
\hline 3.0 & 45 & 2.8 \\
3.4 & 39 & 2.4 \\
3.8 & 39 & 2.4
\end{tabular}

frequency-dependent damping measurements ranging from $1 \mathrm{~Hz}$ to about $70 \mathrm{~Hz}$ (using an increment of $0.5 \mathrm{~Hz}$ ) at a dynamic load of $10 \%$ of $h$ were performed.

Damping results for selected strains or frequencies are shown and displayed as the mean value including a standard deviation taken from three single measurements using three specimens with the same specifications.

In order to investigate the resulting microstructure and consequently the printing process, a microscopic analysis was carried out using a digital microscope (Di-Li 1027-HD, transmitted light mode; Distelkamp-Electronic, Kaiserslautern, Germany). The created pores were completely enclosed by the base material during printing. Transmitted light microscopy was only possible after further samples had been printed, in which the upper and lower sides of the rectangular samples were missing. Only then the pores as well as the immediate surroundings around the pores could be observed in detail. Pictures were taken using an evaluation software adapted to the digital microscope (software "Grab \& Measure"; A-ZYSTEMS, Mainz, Germany).

\section{Results and discussion}

\subsection{Damping measurements}

Figure 5 shows the change in damping at variable pore size or porosity. This is done by analysing the development of the damping $\tan (\delta)$ as a function of the deflection strain $\varepsilon$. Both axes are scaled linearly. Black squares represent the measurements with non-porous samples, while the porous samples are represented by circular symbols. It is common to all measurements that a monotonic increase in damping occurs with the increasing material strain.

Up to a pore size of $1.2 \mathrm{~mm}$, an increase of $\tan (\delta)$ can be seen over the selected deflection strain range from about $4 \%$ to about $17 \%$. Above a pore size of $1.4 \mathrm{~mm}$, a tendency of a reduction of material damping with the increasing pore size can be observed. This indicates that the pore size has an influence on the damping behaviour. However, only larger pores seem to have a significant impact on $\tan (\delta)$.

In Fig. 6, the $\tan (\delta)$ function is plotted versus the deflection strain $\varepsilon$. Analogous to Fig 5, a linear scaling of the axes and the same range of abscissa and ordinate data were chosen. The pore

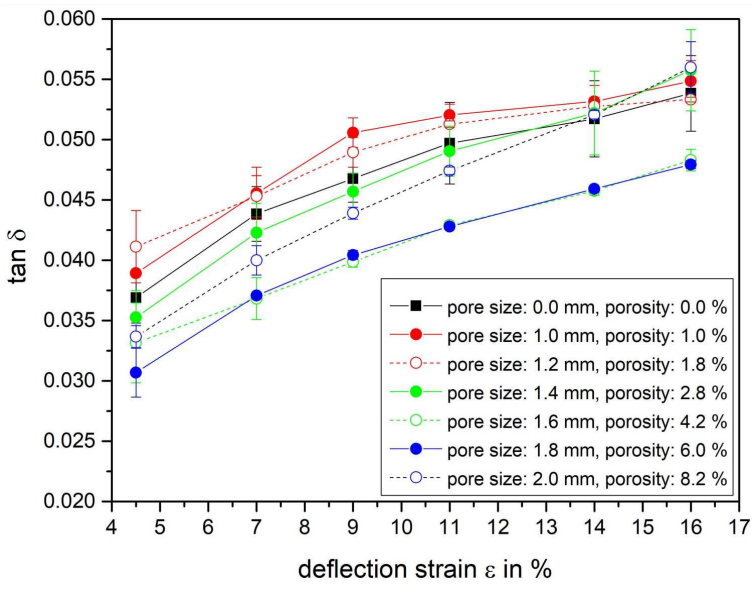

Fig. 5. Strain-dependent damping curves at varying pore sizes.

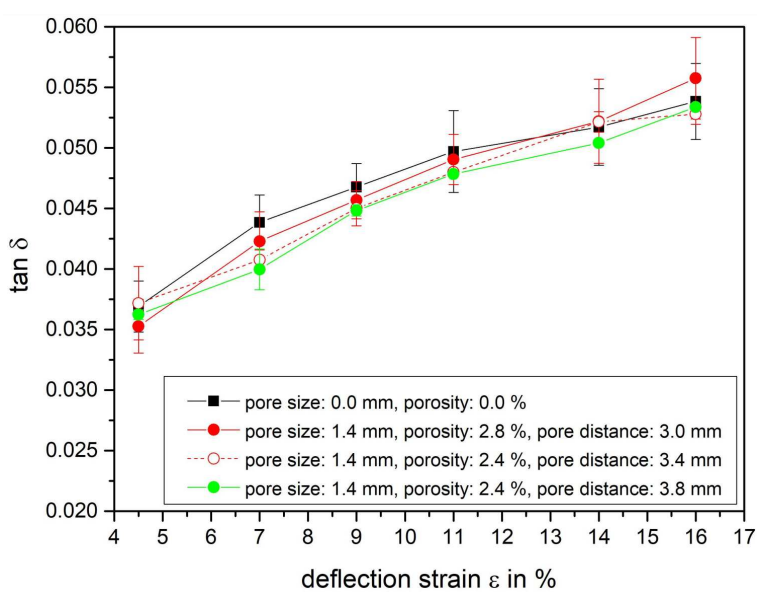

Fig. 6. Strain-dependent damping curves at varying pore distances.

spacing was changed using approximately the same porosity. It must be stressed at this point that the pore spacing was limited in its dimension due to the small sample thickness. Again, a monotonic increase of the damping with the increasing strain was observed. The results of the non-porous samples (black squares) once more serve as a reference. Within the framework of the error bars, it cannot be clearly stated that the pore spacing has a marked influence on the damping behaviour for the same pore size or almost similar porosity.

In order to investigate the dependence of material damping on pore size, further measurements as a function of the frequency were carried out. In Fig. 7, the function dependence of $\tan (\delta)$ versus frequency is shown. Both axes are linearly scaled. The reference measurement on non-porous samples is represented by black squares.

All measurements show a slight decrease in damping with the increasing frequency in the selected frequency range, approximately $10-60 \mathrm{~Hz}$. It can be observed that for a pore size of $1.4-1.8 \mathrm{~mm}$, the damping is below the reference curve. It is 


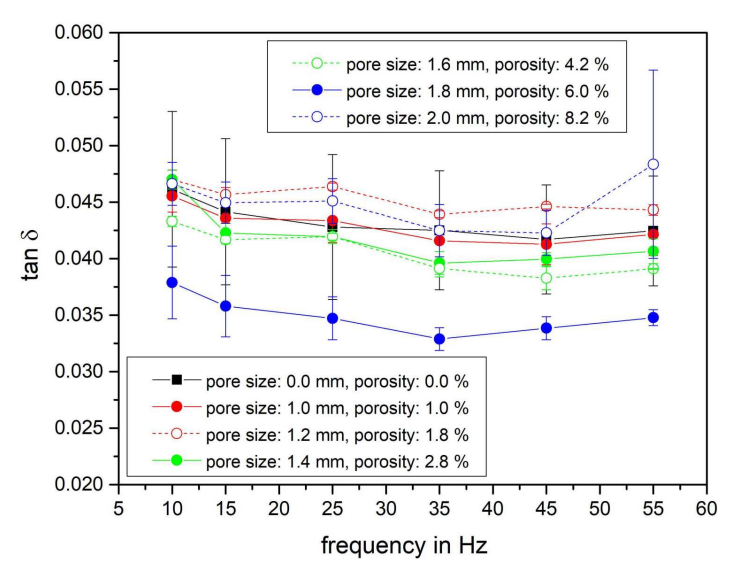

Fig. 7. Frequency-dependent damping curves at varying pore sizes.

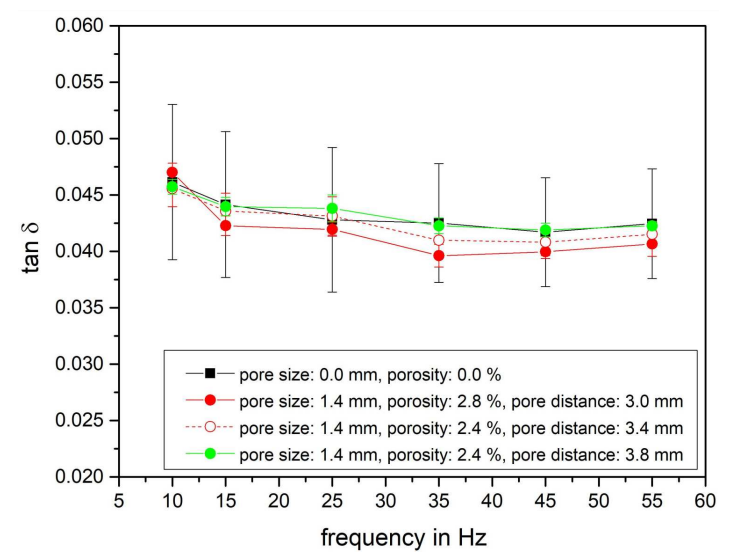

Fig. 8. Frequency-dependent damping curves at varying pore distances.

most pronounced at a pore size of $1.8 \mathrm{~mm}(6.0 \%$ porosity). At a pore size of $2 \mathrm{~mm}$, all damping values are above the reference line. However, it is also noticeable that, the corresponding mean values show the largest standard deviation of all porous samples which are plotted in Fig. 7.

Analogous to the strain-dependent damping measurements, measurements were also carried out with the same pore size or similar porosity but different pore spacing. The reference measurement is also symbolized in this case by black squares.

As seen in Fig. 8, a slight decrease of the damping with the increasing frequency occurs again in all measurements. The data of the non-porous sample have a high standard deviation, so that it is difficult to determine the influence of the pore spacing on the frequency-dependent damping measurement. The comparison of the results of the porous samples gives a hint about a slight increase in material damping with the increasing pore spacing. At the largest pore spacing of $3.8 \mathrm{~mm}$, the curve follows the reference curve. This can be explained by the existence of significantly more material between pores. Therefore, the sample shows a similar behaviour to the non-porous one.

\subsection{Microscopic analysis}

The light microscopy image of Fig. 9 exhibits a sample with a diameter of about $1.3 \mathrm{~mm}$ which should nominally have a diameter of $1.4 \mathrm{~mm}$. In the immediate vicinity around the pores, there are further "voids" (empty spaces) which can vary greatly in both length and width. Later, there will be a distinction between voids of type 1 and of type 2 . In principle, the printer is capable of producing the selected dimensions according to CAD drawings. This becomes clear, among other things, because the pore spacing of $3 \mathrm{~mm}$ (see Fig. 10) was adhered to relatively well. The accuracy of the printer can be estimated at $\pm 0.1 \mathrm{~mm}$. However, the printing process is incomplete and suggests insufficient adhesion of individual filament layers leading to type 1 of voids. This error may become of minor importance with increased pore sizes. However, if the pores have a small diameter, which is in the order of magnitude of the voids, then the damping behaviour is influenced by both the increase in pore size and dry friction (the Coulomb friction). Dry friction is caused by the rubbing of insufficiently bonded filament layers.

Figures 10 and 11 indicate that the sample has a locally varying density due to the existence of some areas with higher transparency than others. Although the sample consists of a fixed network, one can assume that this network contains substructures that are differently pronounced. It is worth

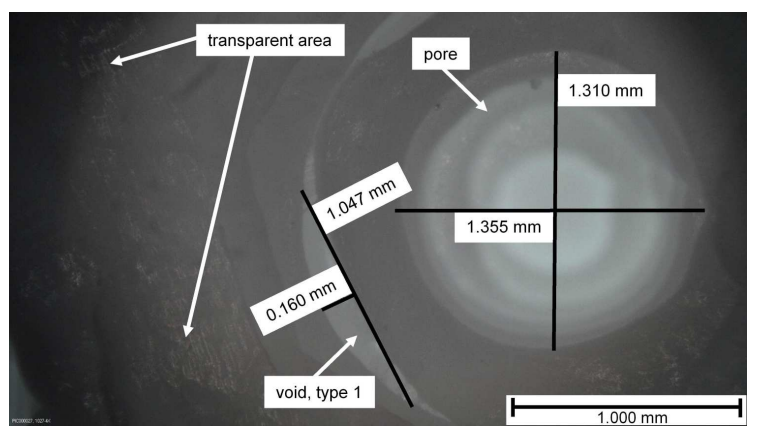

Fig. 9. Light microscopy image; nominal pore size is $1.4 \mathrm{~mm}$.

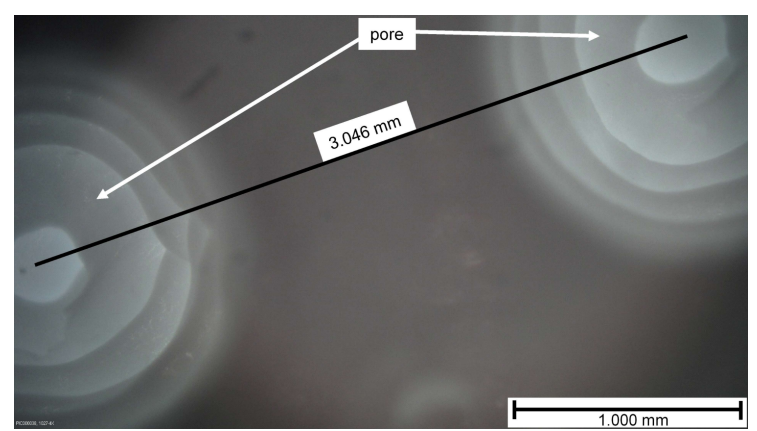

Fig. 10. Light microscopy image; nominal pore distance is $3.0 \mathrm{~mm}$. 


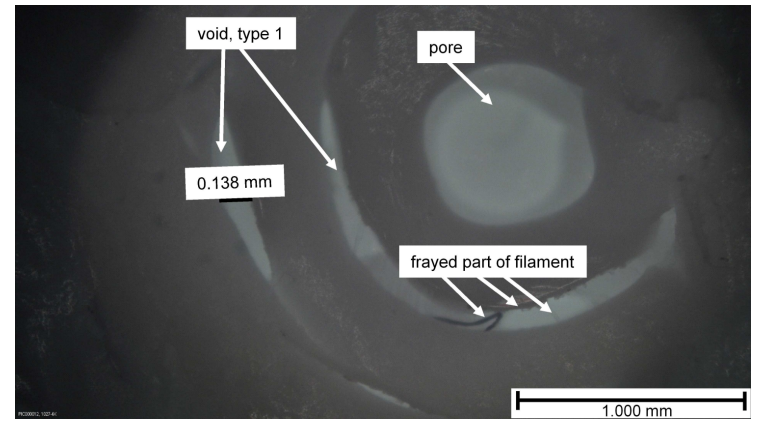

Fig. 11. Light microscopy image; area around the pores.

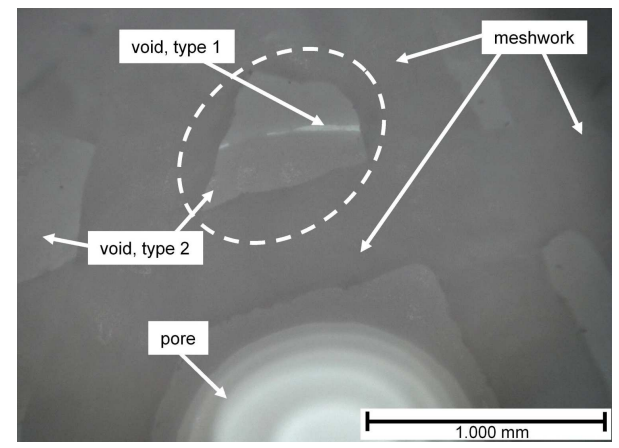

Fig. 12. Light microscopy image; meshwork.

to note that voids (type 1) around the pores are always noticeable. Further, the filament seems to be fraying out (see Fig. 11). These frayed parts of the filament can run through the voids and hence they may be the only connection between adjacent filament layers that have not been bonded in printing.

Beside empty spaces of type 1 , a changed depth of sharpness of the image provides information about the structural composition during the printing process. A scaffolding structure appears as the superordinate structure. Although the filaments may be locally bonded together, they have a mesh type leading to an additional empty space which at this point is to be referred to as the void of type 2. This area is framed with a dashed line in Fig. 12.

It becomes clear now that the sample contains further empty spaces in addition to the pores. These voids are due to thermo-mechanical (type 1) and printer-specific (type 2) causes.

\section{Discussion}

The damping measurements were carried out below the glass transition temperature of PLA which is around $60^{\circ} \mathrm{C}$. PLA may remain mainly amorphous because of its slow crystallisation kinetics [32]. In this regime, bond stretching and bending controls the modulus which directly reflects the stiffness of the Van der Waals bonds which bind one chain to another [33]. A strong stress relaxation and, thus, a strong increase of the damping cannot be expected. side view, upper side

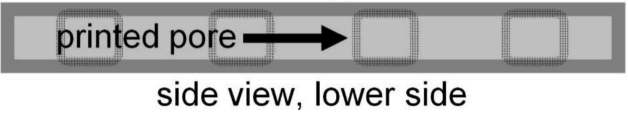

Fig. 13. Side view of the printed samples showing a sandwich structure.

However, the polymer matrix and the pores have different elastic moduli and thermal expansion coefficients. This leads to an inhomogeneous state of stress and strain state [34] which increases when an external oscillating force is applied to the sample. Warping - a common problem in 3D printing usually occurs when the print cools too rapidly [35] and can also contribute to the inhomogeneous state of stress and strain state. As a result, polymer chains within the filament are thus moved and cause a change in the relative position of the chains.

A dependence of damping on the strain, as shown in Figs. 5 and 6 , can be in fact explained by such a motion mechanism. It can also be assumed that the stress field around the pores is intensified by the forced expansion and contraction of the pores resulting from the external load. As a consequence of these mechanisms, an increase in damping with the increasing strain can be observed.

Looking at the printed sample from the side, as shown in Fig. 13, it becomes clear that ultimately a sandwich structure or composite is present. Sandwich panels made of fibre-reinforced plastic skins and a honeycomb core can be effectively damped through the choice of the skin and especially of the core materials [36].

For a sandwich configuration, the total strain and damping energies are assumed to be the sum of separable strain and damping energies of the skins and of the core in each case [36]. The total damping $\tan \left(\delta_{\text {tot }}\right)$ is therefore made up of the damping of the outer area (skin) $\tan \left(\delta_{o}\right)$ and the inner area (core) $\tan \left(\delta_{i}\right)$. This representation is, however, insufficient and the influence of porosity $\tan \left(\delta_{p}\right)$ and cracks $\tan \left(\delta_{c r}\right)$ on the overall damping have to be considered [37]. In this context, the voids of type 1 are considered as cracks. The total damping of a porous material can be expressed as

$$
\tan \left(\delta_{\text {tot }}\right)=\tan \left(\delta_{o}\right)+\tan \left(\delta_{i}\right)+\tan \left(\delta_{p}\right)+\tan \left(\delta_{c r}\right) .
$$

The influence of pores and cracks on the damping was investigated by Arhipov et al. [38] in more detail. In general, the results of damping measurements on porous samples can depend on different damping mechanisms. This has to be taken into account, especially when specimens have been manufactured in a $3 \mathrm{D}$ printing process. The pore size, pore distribution, structure and material of the outer and inner areas of the sample and the quality of bonding between these areas are parameters that make a prediction of material damping considerably more challenging. 


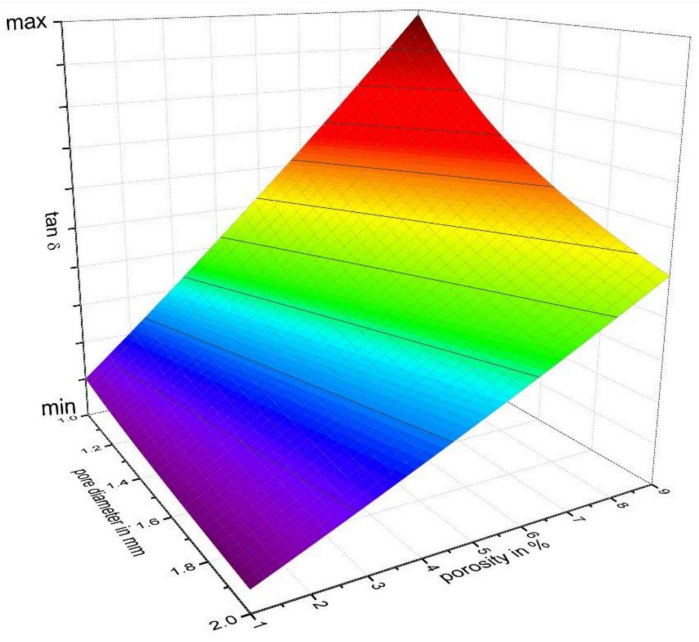

Fig. 14. Damping as a function of the pore diameter and porosity; according to (7).

As reported on metallic foams in [39], very limited information is available about the damping mechanisms in the $\mathrm{Hz}$ and $\mathrm{kHz}$ frequency ranges. The frequency-dependent damping measurements, which are shown in Figs. 7 and 8, exhibit a slight decrease of the damping of the printed samples with the rising frequency. This is valid for non-porous as well as for porous specimens. Taking into account the light microscopy analysis in Fig. 12, it can be stated that the meshwork created by the filaments causes the formation of the voids of type 2 . Therefore, the non-porous sample cannot be characterised as a "dense" one.

In the studies of Wang et al. [40], polyurethane was filled in the voids of a printed lattice structure. Based on the differential equation of vibration of a viscous damped vibration system, the frequencydependent damping of the hybrid composite lattice sandwich truss was determined using a finite element modal calculation. It was found that damping decreases when frequency increases. Since the dynamic behaviour is difficult to assess, the printed material (with or without pores) is to be regarded as a composite against this background. The case of the damping decrease at the rising frequency might be due to reduced movements of molecular chains in the matrix by "physical crosslinks" as proposed by Wei et al. [41]. In particular, the particles dispersed in the matrix can serve as an example of these "physical crosslinks".

According to Wei et al. [42], the pore-influenced damping is writable as

$$
\tan (\delta) \propto \frac{1}{a} \frac{c}{1-c},
$$

where $a$ is the mean radius of the pores and $c$ is the porosity (see (3)). Since the proportionality relationship appears in (5), one can as well write that

$$
\tan (\delta)=\frac{1}{a} \frac{c}{1-c} k
$$

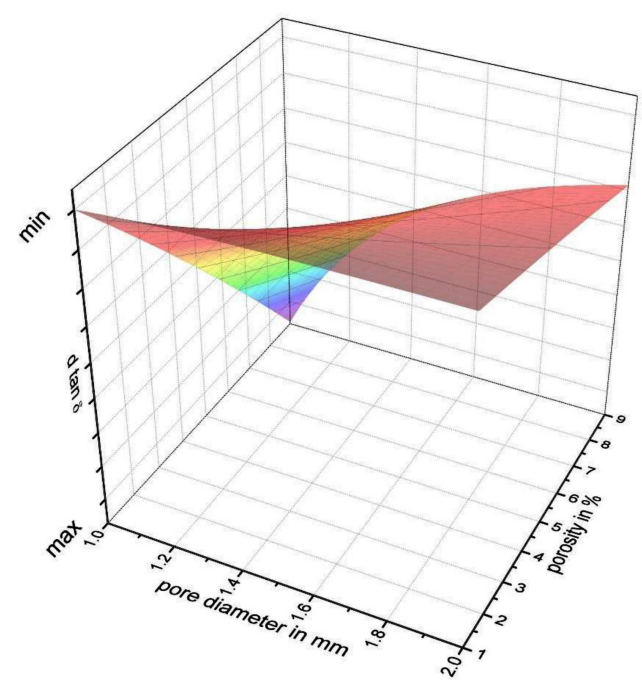

Fig. 15. Calculation of the total differential $\mathrm{d} \tan (\delta)$ to estimate the individual influence of the pore diameter and porosity on the damping; according to (9).

where $k[\mathrm{~mm}]$ is the proportionality factor. In the present studies, the pore size $s$ is considered instead of the pore radius $a$. Therefore, by substituting $a=\frac{s}{2}$, one gets

$$
\tan (\delta)=\frac{2}{s} \frac{c}{1-c} k .
$$

It can be seen that the damping increases with the increasing porosity and decreasing pore diameter (pore size) as well. This behaviour is illustrated in Fig. 14, where $\tan (\delta)$ is plotted as a function of $s$ and $c$. For simplicity, we set $k=1 \mathrm{~mm}$.

However, it is not directly apparent which parameter is most dominant at which point in the $3 \mathrm{D}$ diagram. An analysis of the diagram concerning the individual variables pore diameter and porosity is necessary by calculating the total differential of the function $\tan (\delta)$.

The total differential of a function $f$ describes how the function value $f\left(x_{1}, x_{2}, \ldots, x_{n}\right)$ with independent variables $x_{1}, x_{2}, \ldots, x_{n}$ changes when the variables change infinitesimally at the same time. For the total differential of the function $f$ applies

$$
\mathrm{d} f=\sum_{i=1}^{n} \frac{\partial f}{\partial x_{i}} \mathrm{~d} x_{i}
$$

with $\mathrm{d} f$ being the total differential of the function $f$ and where $\frac{\partial f}{\partial x_{i}}$ is the partial derivation with respect to $x_{i}$ and $\mathrm{d} x_{i}$ is the differential of $x_{i}$. Using (8), we can write that

$$
\mathrm{d} \tan (\delta)=k\left[\frac{2}{s^{2}} \frac{c}{c-1} \mathrm{~d} s+\frac{2}{s} \frac{1}{(c-1)^{2}} \mathrm{~d} c\right] .
$$

Figure 15 shows the total differential of the damping $\mathrm{d} \tan (\delta)$ plotted against the pore diameter and porosity obtained with (9). The selected range of the variables is adapted to the experiment. 
If the pore diameter increases for a given small porosity, the damping changes only slightly. If on the other hand, the porosity is large, even the smallest changes in the pore diameter lead to a strong change in damping. As expected, the maximum damping changes occur at smallest pore diameters and maximum porosity.

One can deduce from the measurements shown here, that a significant change in damping is expected only in the combination of high porosity and small pore size. For this reason, significant differences to the damping results of the non-porous sample only occur with a pore size larger than $1.4 \mathrm{~mm}$ and a porosity larger than $\approx 3 \%$. This can be seen in both the strain-dependent damping measurement (see Fig. 5) and the frequency-dependent damping measurement (see Fig. 7).

Note that in Figs. 6 and 8, the pore size is constant at $1.4 \mathrm{~mm}$ and the porosity does not exceed $2.8 \%$. Both the strain-dependent damping measurement and the frequency-dependent damping measurement do not allow a clear dependence of the damping on both the pore size and porosity for this combination. The progressions of the respective measurements are contradictory because concerning the strain-dependent damping measurement, the lowest damping occurs for the smaller porosity, whereas concerning the frequency-dependent damping measurement, this case is evident for the larger porosity. It is assumed that the influence of other damping mechanisms predominates for this combination of pore size and porosity.

\section{Conclusions}

Cellular materials play an important role in vibration and noise reduction. Using a dynamic mechanical analyser (DMA), the damping as a function of strain and frequency was investigated on samples produced using a standard 3D printer. The samples differed in pore size and porosity. It was observed that the measuring method is very sensitive to changes in the microstructure of the samples and therefore has enormous potential for the characterization of the sample structure.

Both the strain-dependent and the frequencydependent damping measurements on the reference sample, which was initially considered to be free of pores, showed a strong scattering of the measurement results. Light microscopy analysis of the printed samples gave a hint that the quasi-real sample already contains empty spaces (voids of type 1). These are caused, among other things, by insufficient bonding of the filaments to each other. In addition, the laying of the filament strands caused by the printer can lead to a superordinate skeleton structure (meshwork) which also contains further empty spaces (the voids of type 2). The voids of type 1 are assumed to be acting as cracks.

This special meshwork activates further damping mechanisms which reduce the influence of pore size or porosity on the strain-dependent or frequencydependent damping behaviour. Only from a certain combination of pore size and porosity onwards, a dominant influence of these two parameters can apparently be expected.

\section{Acknowledgments}

Prof. Dr. Jürgen Göken gratefully acknowledges the financial support of the German Research Foundation (DFG, www.dfg.de; DFG-reference number: INST 21572/5-1 FUGG).

The authors would also like to express their gratitude to Master's student Mrs. Brinkmann for her scientific assistance and research associate Mr. Stampa for the sample preparation.

\section{References}

[1] V. Mazzanti, L. Malagutti, F. Mollica, Polymers 11, 1094 (2019).

[2] Y.-C. Wang, T. Chen, Y.-L. Yeh, Int. J. Adv. Manufact. Technol. 105, 4059 (2019).

[3] A. Nuseir, M.M. Hatamleh, A. Alnazzawi, M. Al-Rabab'ah, B. Kamel, E. Jaradat, J. Prosthodont. 28, 10 (2019).

[4] A. Lee, A.R. Hudson, D.J. Shiwarski, J.W. Tashman, T.J. Hinton, S. Yerneni, J.M. Bliley, P.G. Campbell, A.W. Feinberg, Science 365, 482 (2019).

[5] H. Jeong, S.-J. Han, S.-H. Choi, Y.J. Lee, S.T. Yi, K.S. Kim, Materials 12, 657 (2019).

[6] A. Guo, H. Li, J. Xu, J. Li, F. Li, e-Polymers 20, 103 (2020).

[7] U.G.K. Wegst, Am. J. Bot. 93, 1439 (2006).

[8] P. Göransson, Philos. Trans. R. Soc. A 364, 89 (2006).

[9] N. Rako-Gospić, M. Picciulin, Underwater Noise: Sources and Effects on Marine Life, in: World Seas: An Environmental Evaluation, Vol. III, Ed. C. Sheppard, Academic Press, London 2019, Ch. 20, p. 367.

[10] N. Shahrubudin, T.C. Lee, R. Ramlan, Proced. Manufact. 35, 1286 (2019).

[11] J. Banhart, J. Baumeister, M. Weber, Mater. Sci. Eng. A 205, 221 (1996).

[12] I.S. Golovin, H.-R. Sinning, Mater. Sci. Eng A 370, 504 (2004).

[13] I.S. Golovin, H.-R. Sinning, J. Göken, W. Riehemann, Solid State Phenom. 89, 267 (2003).

[14] F. Han, Z. Zhu, C. Liu, J. Gao, Metall. Mater. Trans. A 30, 771 (1999).

[15] B.J. Lazan, Damping of Materials and Members in Structural Mechanics, Pergamon Press, Oxford, UK 1968. 
[16] K.S. Kwan, Ph.D. Thesis, Virginia Polytechnic Institute and State University, Blacksburg, USA 1998.

[17] J. Göken, S. Fayed, H. Schäfer, J. Enzenauer, Acta Phys. Pol. A 133, 1241 (2018).

[18] T.R. Tiersch, W.T. Monroe, Cryobiology 73, 396 (2016).

[19] K. Van de Velde, P. Kiekens, Polym. Test. 21, 433 (2002).

[20] S. Farah, D.G. Anderson, R. Langer, Adv. Drug Deliv. Rev. 107, 367 (2016).

[21] G.H. Yew, A.M. Mohd Yusof, Z.A. Mohd Ishak, U.S. Ishiaku, Polym. Degrad. Stabil. 90, 488 (2005).

[22] G.Y.H. Choong, A.J. Parsons, D.M. Grant, D.S.A. De Focatiis, in: AIP Conf. Proc. 1664, 170005 (2015).

[23] F. Asghari, M. Samiei, K. Adibkia, A. Akbarzadeh, S. Davaran, Artif. Cell Nanomed. B 45, 185 (2017).

[24] Z. Samouh, K. Molnar, F. Boussu, O. Cherkaoui, R. El Moznine, Polym. Adv. Technol. 30, 529 (2019).

[25] Velleman, $1.75 \mathrm{~mm}(1 / 16 ")$ PLA filament, access on 22nd May 2020.

[26] All3DP, Creality CR-20 printer review, access on 25th May 2020.

[27] A. Dey, N. Yodo, J. Manuf. Mater. Process. 3, 64 (2019).

[28] A. Rasa, in: Proc. 43rd Int. Congress and Exposition on Noise Control Engineering - Improving the World through Noise Control, Melbourne 2014, Eds. J. Davy, T. McMinn, N. Broner, C. Don, L. Dowsett, M. Burgess, Internoise, 2014, p. 4278 .
[29] K.P. Menard, Dynamic Mechanical Analysis, A Practical Introduction, CRC Press, Boca Raton (FL) 1999.

[30] Netzsch Gabo Instruments GmbH homepage, access on 25th May 2020.

[31] M. Eragubi, Int. J. Innov. Manag. Technol. 4, 410 (2013).

[32] O. Martin, L. Avérous, Polymer 42, 6209 (2001).

[33] D.G. Gilbert, M.F. Ashby, P.W.R. Beaumont, J. Mater. Sci. 21, 3194 (1986).

[34] G. Laplanche, P. Gadaud, C. Bärsch, K. Demtröder, C. Reinhart, J. Schreuer, E.P. George, J. Alloys Compd. 746, 244 (2018).

[35] P. Stavropoulos, P. Foteinopoulos, A. Papacharalampopoulos, G. Tsoukantas, Int. J. Adv. Manuf. Technol. 104, 1571 (2019).

[36] A. Abbasloo, M.R. Maheri, Sci. Eng. Compos. Mater. 25, 649 (2018).

[37] J. Göken, W. Riehemann, Mater. Sci. Eng. A 370, 417 (2004).

[38] I.K. Arhipov, I.S. Golovin, S.A. Golovin, H.-R. Sinning, Philos. Mag. 85, 1557 (2005).

[39] I.S. Golovin, H.-R. Sinning, Solid State Phenom. 89, 261 (2003).

[40] R. Wang, J. Shang, X. Li, Z. Luo, W. Wu, Sci. Rep. 8, 9604 (2018).

[41] W. Wei, Y. Zhang, M. Liu, Y. Zhang, Y. Yin, W.S. Gutowski, P. Deng, C. Zheng, Polymers 11, 647 (2019).

[42] J.N. Wei, H.F. Cheng, C.L. Gong, F.S. Han, J.P. Shui, Metall. Mater. Trans. A 33, 3565 (2002). 